\title{
TMPRSS3 modulates ovarian cancer cell proliferation, invasion and metastasis
}

\author{
DAN ZHANG, SHUANG QIU, QI WANG and JIANHUA ZHENG \\ Department of Obstetrics and Gynecology, The First Affiliated Hospital of Harbin Medical University, \\ Harbin, Heilongjiang 150001, P.R. China
}

Received June 3, 2015; Accepted July 13, 2015

DOI: 10.3892/or.2015.4356

\begin{abstract}
Overexpression of transmembrane protease, serine 3 (TMPRSS3) has been detected in ovarian cancer. However, the molecular mechanisms of TMPRSS3 in ovarian cancer remain unclear. In the present study, we found that TMPRSS3 was significantly expressed in ovarian cancer cells. Overexpression of TMPRSS3 promoted the proliferation, invasion and migration of A2780 cells. Conversely, knockdown of TMPRSS3 in HO8910 cells inhibited the proliferation, invasion and migration. Furthermore, TMPRSS3 affected the expression levels of E-cadherin, vimentin and Twist. In addition, TMPRSS3 induced activation of ERK1/2 in ovarian cancer cells, and the ERK1/2 pathway was required for the TMPRSS3-mediated proliferation, invasion and migration of ovarian cancer cells. Finally, knockdown of TMPRSS3 inhibited ovarian cancer HO8910 cell growth and metastasis in vivo. Collectively, the present study suggests that TMPRSS3 plays a crucial role in the development and progression of ovarian cancer. Therefore, TMPRSS3 represents a potential therapeutic target of ovarian cancer.
\end{abstract}

\section{Introduction}

Ovarian cancer is one of the most common gynecologic malignancies worldwide, and is a leading cause of cancer-related death among women (1). The overall 5-year survival of earlystage ovarian cancer patients is higher than $65 \%$. However, advanced ovarian cancer develops resistance to chemotherapy and radiotherapy, and eventually leads to death (2). Therefore, the search for novel biomarkers for the early diagnosis of ovarian cancer is urgently needed.

Correspondence to: Dr Jianhua Zheng, Department of Obstetrics and Gynecology, The First Affiliated Hospital of Harbin Medical University, 23 You Zheng Road Harbin, Heilongjiang 150001, P.R. China

E-mail: jianhuazheng12@126.com

Key words: TMPRSS3, proliferation, invasion, metastasis, ERK1/2 pathway, ovarian cancer
Extracellular proteolytic enzymes, including matrix metalloproteinases (MMPs) and serine proteases, have been found to contribute to tumor cell invasion and metastasis $(3,4)$. Recently, type II transmembrane serine proteases (TTSPs) have been recognized as a new subfamily of the serine proteases $(5,6)$. Numerous TTSPs are highly expressed in cancer, and have been implicated in tumor development and progression (4). Belonging to the TTSP family, the TMPRSS subfamily consists of four members, TMPRSS2-5. As one member of the TMPRSS family, transmembrane protease, serine 3 (TMPRSS3) expression has been detected in a number of human tissues, including the heart, kidney, liver, lung and ovary (7). TMPRSS3 has been found to be overexpressed in numerous types of cancers including pancreatic, gastric and colon cancer (8). Microarray of pancreatic cancer has identified that TMPRSS3 is one of the most differentially expressed genes, and acts as a tumor marker for screening pancreatic cancer (9). It has been reported that the TMPRSS3 expression level in ovarian carcinomas is elevated, and may be useful as a molecular target for diagnosis and therapeutic intervention in ovarian cancer. However, the molecular mechanisms of TMPRSS3 in ovarian cancer remain unclear.

In the present study, we investigated the expression of TMPRSS3 in ovarian cancer cell lines. Moreover, we explored the effects of TMPRSS3 expression on the proliferation, invasion and metastasis of ovarian cancer cells. We also aimed to elucidate the molecular mechanisms of TMPRSS3 in ovarian cancer. These data may provide information for the diagnosis of ovarian cancer.

\section{Materials and methods}

Reagents. Antibodies against TMPRSS3, E-cadherin, vimentin, Twist and $\beta$-actin were obtained from Santa Cruz Biotechnology (Santa Cruz, CA, USA). Antibodies against ERK1/2 and phosho-ERK1/2 were obtained from Cell Signaling Technology (Danvers, MA, USA). ERK1/2 special inhibitor U0126 was purchased from Sigma (St. Louis, MO, USA).

Cell lines and culture condition. Human ovarian surface epithelial cell line IOSE144 and human ovarian cancer cell lines A2780, OVCAR3, SKOV3 and HO8910 were all purchased from the Cell Bank of the Chinese Academy of 
Science (Shanghai, China). The IOSE144 cell line was maintained in MCDB105 medium containing 10\% fetal bovine serum (FBS). A2780 and SKOV3 cell lines were maintained in Dulbecco's modified Eagle's medium (DMEM) containing 10\% FBS, while OVCAR3 and HO8910 cell lines were maintained in RPMI-1640 medium containing 10\% FBS. All cell lines were incubated in a $\mathrm{CO}_{2}$ incubator at $37^{\circ} \mathrm{C}$.

Cell transfection. To upregulate the expression of TMPRSS3 in A2780 cells, the human TMPRSS3 full length cDNA was amplified and inserted into the pcDNA3.1 vector (GenePharma Co., Ltd., Shanghai, China) to obtain pcDNA3.1-TMPRSS3, and a scramble sequence was inserted into the pcDNA3.1 vector as the control vector. To knock down the expression of TMPRSS3 in HO8910 cells, a TMPRSS3 shRNA was designed and obtained from GenePharma Co., Ltd. A scrambled shRNA was used as the control shRNA. For transfection, the cells were seeded into 6-well plates. When cell confluency reached 50\%, TMPRSS3 shRNA or pcDNA3.1-TMPRSS3 was transfected into the cells using Lipofectamine 2000 transfection reagent (Invitrogen) according to the manufacturer's instructions. Culture medium was replaced after $6 \mathrm{~h}$ of incubation. Finally, the stable transfected cells were selected by G418 selection and the efficiency was determined by western blotting.

Western blotting. Cells were lysed in RIPA buffer with protease and phosphatase inhibitors, and then the concentration of protein was determined by the BCA assay. Equal amount of protein samples was loaded onto $10 \%$ sodium dodecyl sulfatepolyacrylamide gel electrophoresis (SDS-PAGE) and was then transferred to PVDF membranes. After blocking with 5\% BSA for $1 \mathrm{~h}$ at room temperature, the membranes were incubated with primary antibodies specific to TMPRSS3, E-cadherin, vimentin, Twist, $\beta$-actin, ERK $1 / 2$ or phosho-ERK $1 / 2$ at $4{ }^{\circ} \mathrm{C}$ overnight. Then, the membranes were incubated with horseradish peroxidase-conjugated secondary antibody (Zhongshan Biotech Co., Ltd, Beijing, China) for $1 \mathrm{~h}$ at room temperature. The protein bands were visualized by enhanced chemiluminescence (Applygen Technologies Inc., Beijing, China) and were densitometrically analyzed by Quantity One software (Bio-Rad, Hercules, CA, USA).

Proliferation assay. Cell proliferation was determined by the CCK-8 assay (Jingmei Biotech, Shanghai, China). In brief, the cells were seeded at 1,000 cells/well in a 96-well plate. After incubation with U0126 or dimethylsulfoxide (DMSO) for the indicated time, $10 \mu \mathrm{l}$ of CCK-8 was added into the plate and incubated for $2 \mathrm{~h}$. The optical density (OD) was measured by a microplate reader (Bio-Rad Model 680) at $490 \mathrm{~nm}$ wavelength. The OD value was measured every day for seven days.

In vitro invasion assay. A 24-well Transwell chamber (Corning Costar, New York, NY, USA), which was covered with $30 \mu 1$ of Matrigel (BD Biosciences, USA) to create an artificial basement membrane, was used to examine the invasive ability of the ovarian cancer cells. Cells $\left(5 \times 10^{5}\right)$ were suspended in $200 \mu \mathrm{l}$ serum-free 1640 medium and were added into the upper Transwell chamber. The lower Transwell chamber was filled with $600 \mu \mathrm{l}$ of 1640 medium supplemented with $20 \%$ FBS. After incubation of $16 \mathrm{~h}$ at $37^{\circ} \mathrm{C}$, the non-invading cells

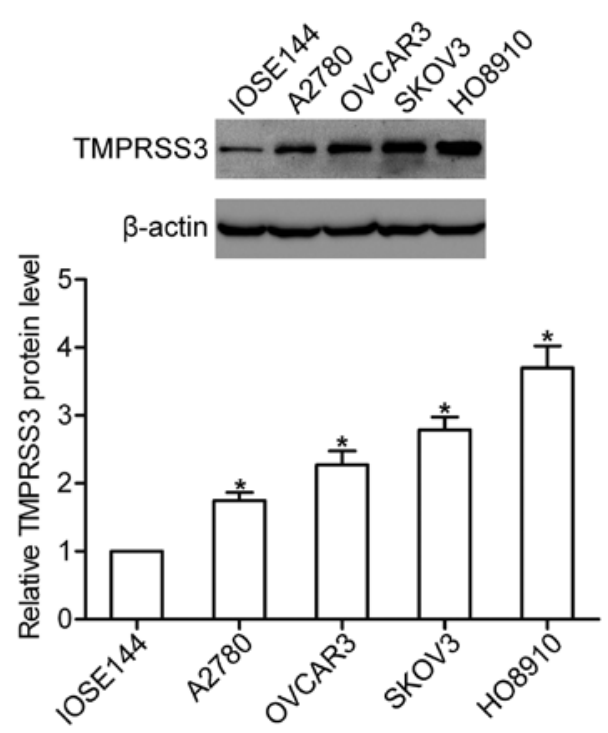

Figure 1. Expression of TMPRSS3 in ovarian cancer cells. The protein level of TMPRSS3 in human ovarian surface epithelial cells (IOSE144) and ovarian cancer cells (A2780, OVCAR3, SKOV3 and HO8910) was detected by western blotting. Data are expressed as the mean $\pm \mathrm{SD}$ of three independent experiments, ${ }^{\mathrm{p}}<0.05$.

were removed with a sterile cotton swab, and the invaded cells were stained with $0.1 \%$ crystal violet for $20 \mathrm{~min}$ at room temperature. The numbers of cells were calculated under a light microscope in five random fields.

In vitro migration assay. Migratory ability was determined using a 24-well Transwell chamber (Corning Costar) not covered with Matrigel. Cells ( $1 \times 10^{5}$ cells/200 $\mu 1$ serum-free 1640 medium) were added into the upper Transwell chamber, and $600 \mu \mathrm{l}$ of 1640 medium supplemented with $20 \%$ FBS was added into the lower Transwell chamber. After incubation of $16 \mathrm{~h}$ at $37^{\circ} \mathrm{C}$, the non-migrating cells were removed with a sterile cotton swab, and the migrated cells were stained with $0.1 \%$ crystal violet for $20 \mathrm{~min}$ at room temperature. The numbers of cells were calculated under a light microscope in five random fields.

In vivo growth and metastasis experiments. Female BABL/c nude mice, four weeks old, were maintained in pathogen-free conditions, and in vivo growth and metastasis experiments were performed after approval by the Animal Care and Use Committee of Harbin Medical University. Mice were subcutaneously injected with $1.0 \times 10^{5}$ cells at the back $(n=8$ for each group). Tumors were observed after one week. Then the length (L) and width $(\mathrm{W})$ of the tumors in mice were measured every week, and the tumor volumes were estimated with the formula: $0.52 \times \mathrm{L} \times \mathrm{W}^{2}$. Seven weeks later, the mice were sacrificed and the livers were fixed in $4 \%$ paraformaldehyde, sectioned into slices and were then stained with hematoxylin and eosin (H\&E). Finally, every slice was observed under a microscope, and the number of micrometastases was counted.

Statistical analysis. Each experiment was performed at least three times. Data are expressed as the mean \pm standard deviation (SD). SPSS 17.0 software (SPSS, Inc., Chicago, IL, USA) was used to perform statistical analysis. Differences between 
A

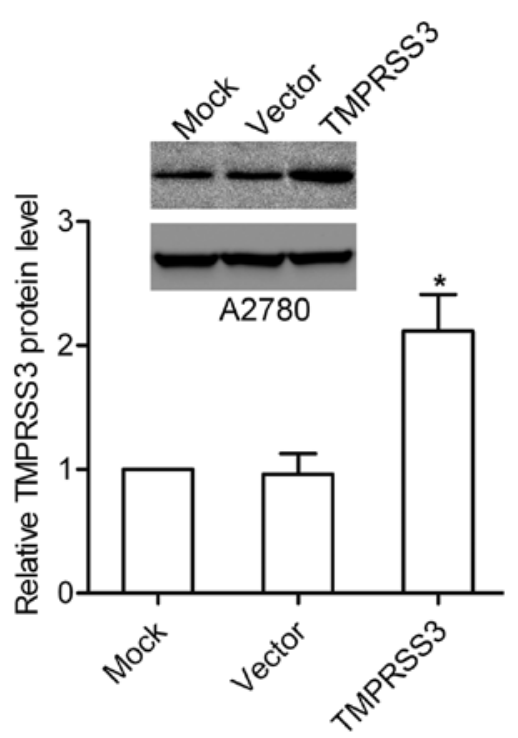

C

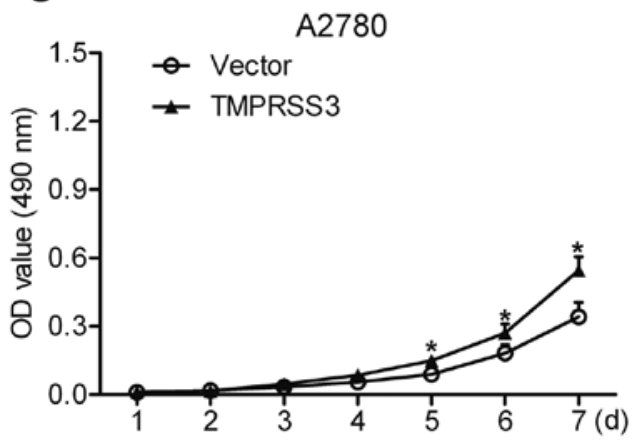

B

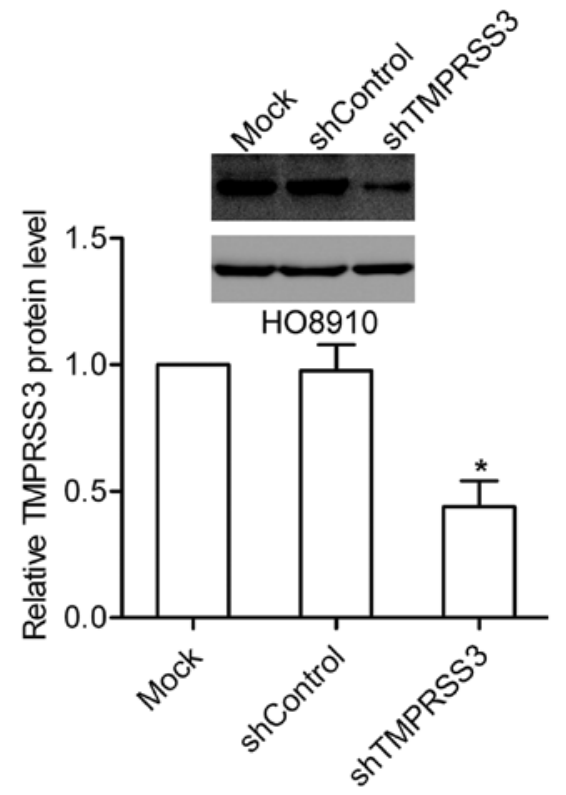

D

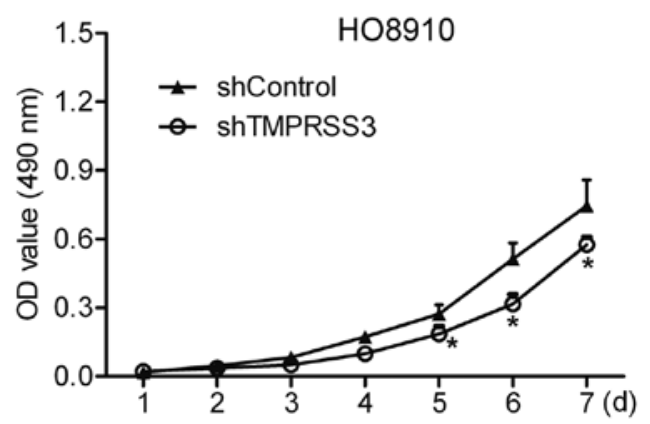

Figure 2. Effect of TMPRSS3 expression on ovarian cancer cell proliferation. (A) A2780 cells were transfected with pcDNA3.1-TMPRSS3 or the pcDNA3.1-vector, and the expression of TMPRSS3 was observed by western blotting. (B) HO8910 cells were transfected with the TMPRSS3 shRNA or control shRNA, and the expression of TMPRSS3 was detected by western blotting. (C) Effect of TMPRSS3 overexpression on the proliferation of A2780 cells. (D) Effect of TMPRSS3 knockdown on the proliferation of HO8910 cells. Data are expressed as the mean \pm SD of three independent experiments, ${ }^{*} \mathrm{p}<0.05$.

groups were evaluated by the Student's t-test or one-way ANOVA. $\mathrm{p}<0.05$ was considered to indicate a statistically significant result.

\section{Results}

Expression of TMPRSS3 in ovarian cancer cells. We first detected the protein level of TMPRSS3 in human ovarian surface epithelial (IOSE144) and ovarian cancer cells (A2780, OVCAR3, SKOV3 and HO8910) by western blotting. The results showed that TMPRSS3 was highly expressed in all ovarian cancer cell lines. A higher protein level of TMPRSS3 was observed in the HO8910 cells, whereas the expression of TMPRSS3 expression was weak in the A2780 cells (Fig. 1).

TMPRSS3 participates in the proliferation of ovarian cancer cells in vitro. To investigate the biological functions of TMPRSS3 in ovarian cancer cells, A2780 cells were transfected with pcDNA3.1-TMPRSS3 to increase TMPRSS3 expression (Fig. 2A), and HO8910 cells were transfected with TMPRSS3 shRNA to deplete endogenous TMPRSS3 expression (Fig. 2B). Cells transfected with the scrambled pcDNA3.1 or shRNA were used as a negative control. The proliferation assay was then performed to explore the effect of TMPRSS3 expression on ovarian cancer cell proliferation. The results showed that overexpression of TMPRSS3 promoted the proliferation of A2780 cells, whereas knockdown of TMPRSS3 led to a decrease in the growth of HO8910 cells (Fig. 2C and D), indicating the involvement of TMPRSS3 in regulating ovarian cancer cell proliferation.

TMPRSS3 is involved in the invasion and migration of ovarian cancer cells in vitro. We further examined the effect of TMPRSS3 expression on ovarian cancer cell invasion and migration. Using invasion and migration assays, we found that the invasive and migratory abilities of the TMPRSS3-expressing A2780 cells were significantly higher when compared with the vector-transfected cells (Fig. 3A and B). In contrast, knockdown of TMPRSS3 attenuated the invasion and migration of the HO8910 cells (Fig. 3C and D). These data suggest that TMPRSS3 plays a pivotal role in the regulation of ovarian cancer cell invasion and migration.

TMPRSS3 affects invasion-related genes in ovarian cancer cells. Numerous studies have revealed that the expression levels of various genes, including E-cadherin, vimentin and 

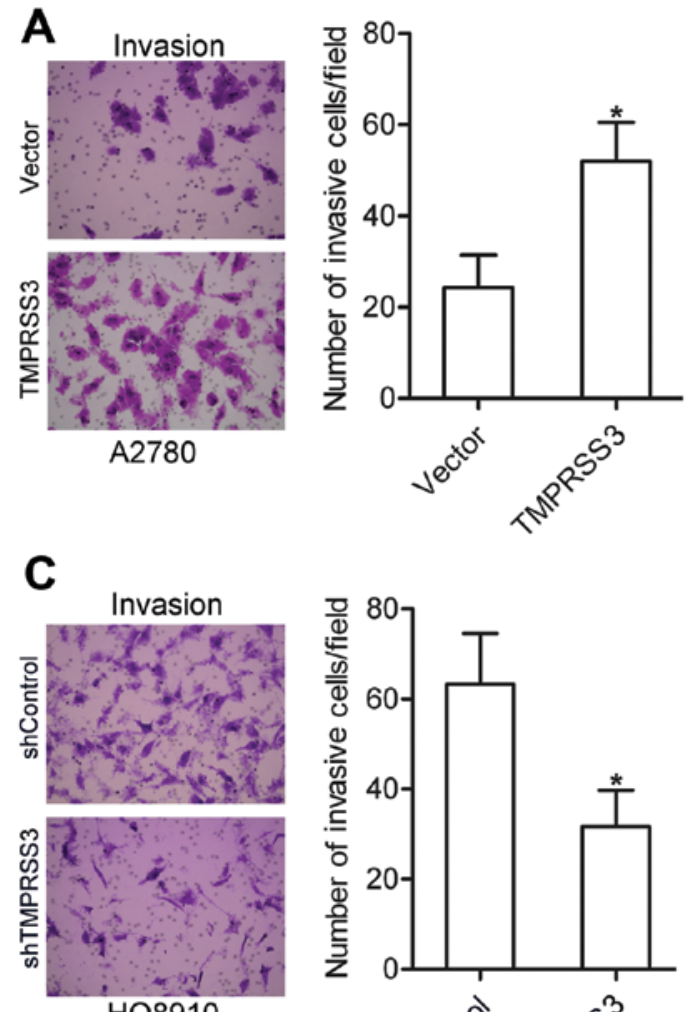

$\mathrm{HO} 8910$

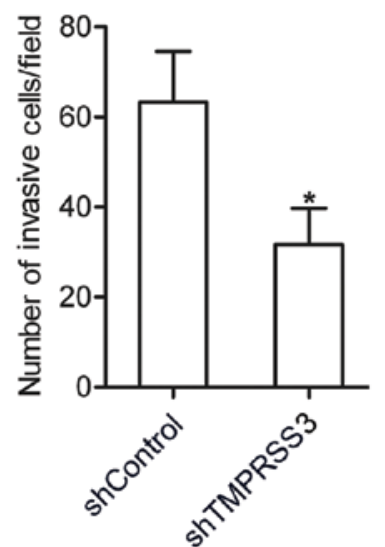

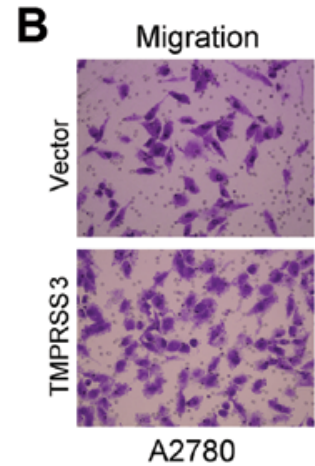

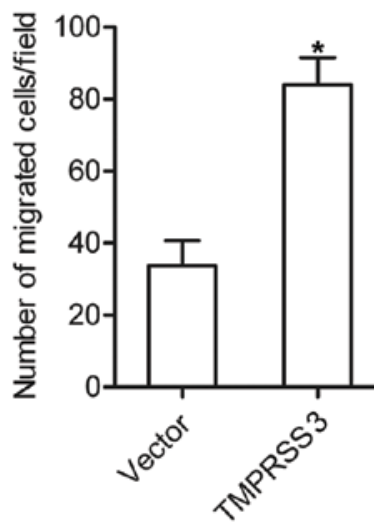

D
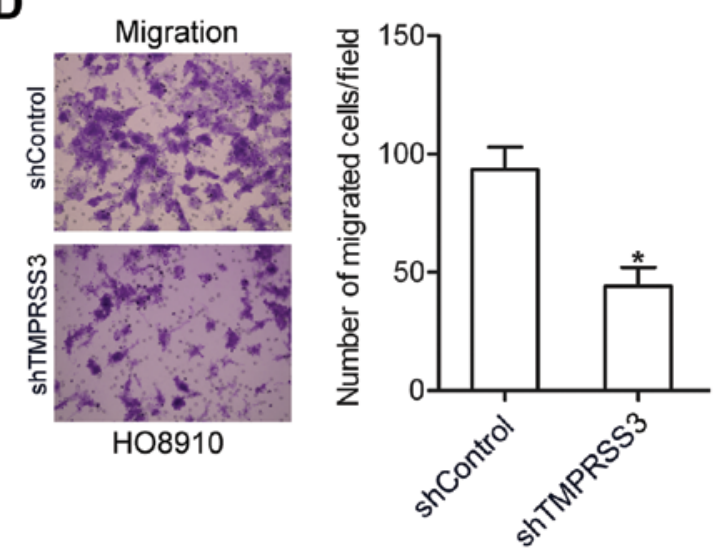

Figure 3. Effects of TMPRSS3 expression on the invasion and migration of ovarian cancer cells. (A and B) Effects of TMPRSS3 overexpression on the invasion and migration of A2780 cells. (C and D) Effect of TMPRSS3 knockdown on the invasion and migration of HO8910 cells. Data are expressed as the mean \pm SD of three independent experiments, $\mathrm{p}<0.05$.

Twist are associated with tumor invasion and metastasis (10). In the present study, we found that overexpression of TMPRSS3 resulted in decreased E-cadherin expression and elevated vimentin and Twist expression in the A2780 cells. In contrast, knockdown of TMPRSS3 led to increased expression of E-cadherin and decreased expression of vimentin and Twist in the HO8910 cells (Fig. 4A-C).

TMPRSS3 induces activation of the ERK1/2 pathway. ERK1/2, a key player in the MAPK signaling pathway, is essential for tumor progression (11). In the present study, we detected the effect of TMPRSS3 expression on the activation of ERK1/2 by western blotting. As shown in Fig. 5, overexpression of TMPRSS3 stimulated the phosphorylation of ERK1/2 in the A2780 cells, whereas knockdown of TMPRSS3 suppressed the phosphorylation of ERK1/2 in the HO8910 cells.

TMPRSS3 contributes to ovarian cancer cell proliferation and invasion via the ERK1/2 pathway. To determine the role of the ERK1/2 pathway in the TMPRSS3-mediated proliferation and invasion of ovarian cancer cells, a special ERK1/2 inhibitor U0126 $(20 \mu \mathrm{M})$ was used to block the activation of ERK1/2. The results showed that overexpression of TMPRSS3 promoted the proliferation, invasion and migration of A2780 cells. However, after inhibition of the ERK1/2 pathway, the proliferation, invasion and migration mediated by TMPRSS3 expression were markedly suppressed (Fig. 6A-C).
These results indicate that the ERK1/2 pathway is involved in the TMPRSS3-mediated proliferation, invasion and migration of ovarian cancer cells.

The ERK1/2 pathway is required for TMPRSS3-mediated expression of E-cadherin, vimentin and Twist. We further detected the role of the ERK1/2 pathway in the TMPRSS3mediated expression of E-cadherin, vimentin and Twist. Notably, we found that U0126 treatment attenuated the TMPRSS3-mediated expression changes in E-cadherin, vimentin and Twist in the A2780 cells (Fig. 7A-C). These data suggest that TMPRSS3 regulates the expression of E-cadherin, vimentin and Twist via the ERK1/2 pathway in ovarian cancer cells.

Knockdown of TMPRSS3 suppresses ovarian cancer cell growth and metastasis in vivo. In vivo growth and metastasis experiments were performed to detect the effect of TMPRSS3 on cell growth and metastasis in vivo. shControl and shTMPRSS3 cells were subcutaneously injected in the back of the mice, respectively. Growth analysis showed that knockdown of TMPRSS3 suppressed ovarian cancer HO8910 cell growth (Fig. 8A), suggesting that TMPRSS3 regulates the growth of ovarian cancer cells in vivo. In addition, liver micrometastasis in the section was counted under a microscope. The results showed that the number of micrometastases was greatly decreased in the shTMPRSS3 group as compared 
A
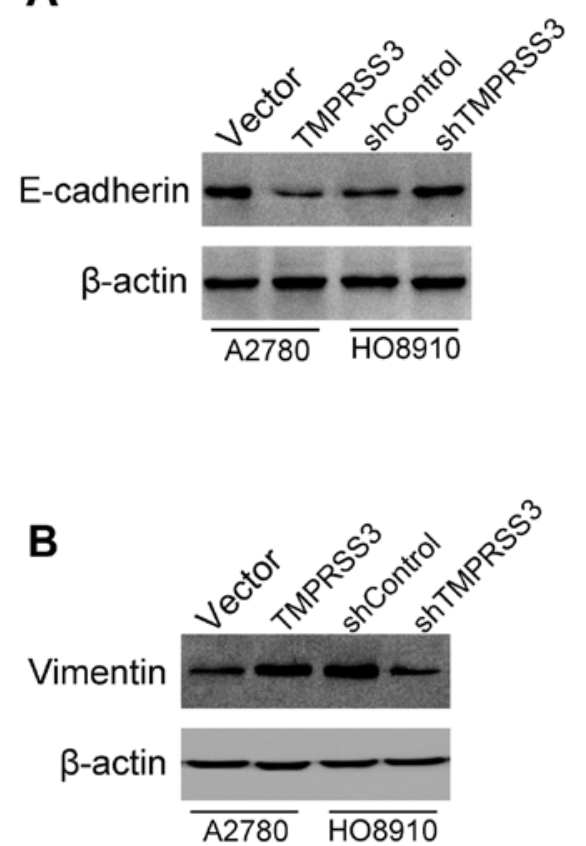

C

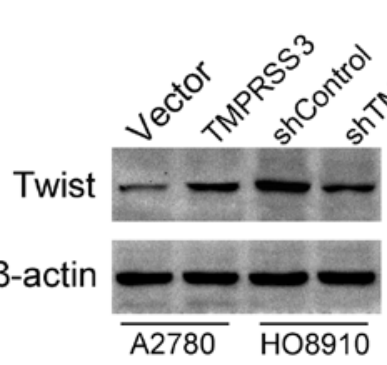

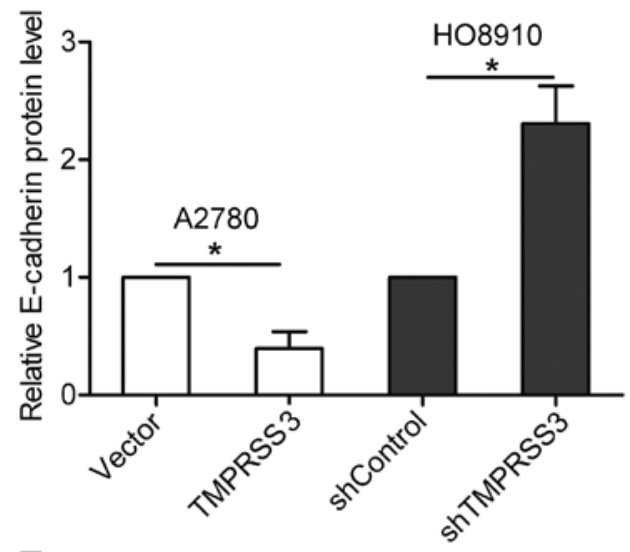
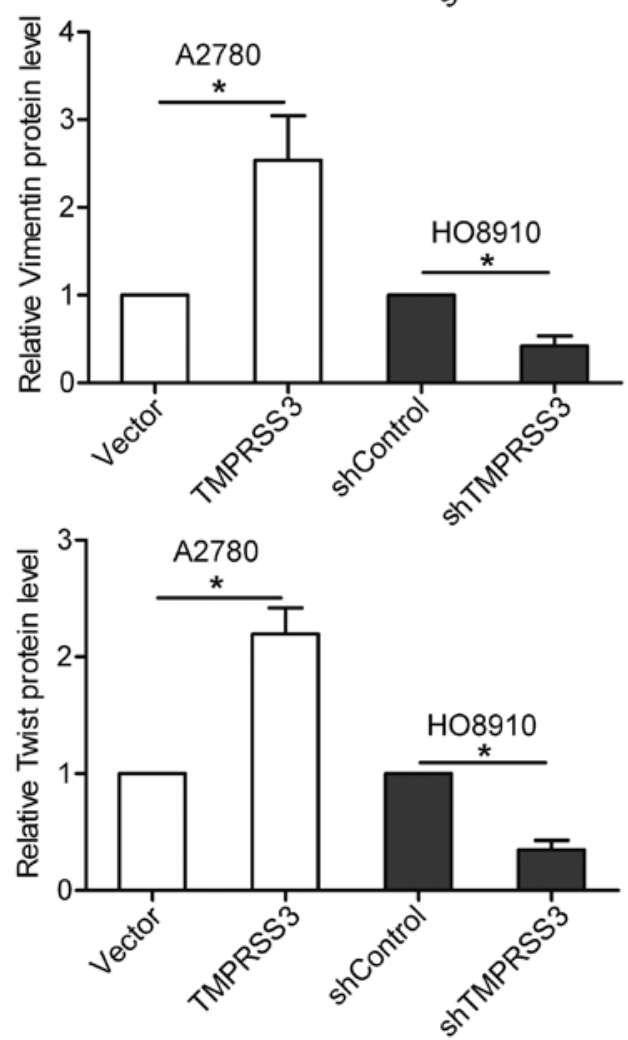

Figure 4. Influence of TMPRSS3 on the expression levels of various invasion-related genes. (A) Effect of TMPRSS3 on the expression of E-cadherin. (B) Effect of TMPRSS3 on the expression of vimentin. (C) Effect of TMPRSS3 on the expression of Twist. Data are expressed as the mean \pm SD of three independent experiments, ${ }^{*} \mathrm{p}<0.05$.
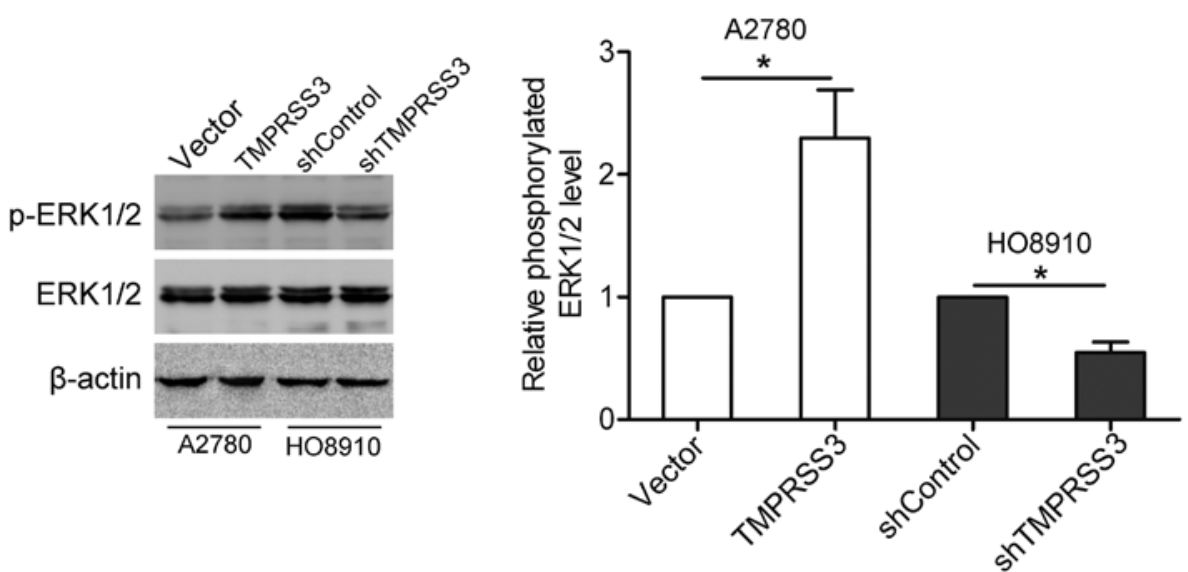

Figure 5. Effect of TMPRSS3 expression on activation of ERK1/2. The phosphorylation of ERK1/2 in A2780 and HO8910 cells was detected by western blotting. Data are expressed as the mean \pm SD of three independent experiments, ${ }^{*} \mathrm{p}<0.05$ 


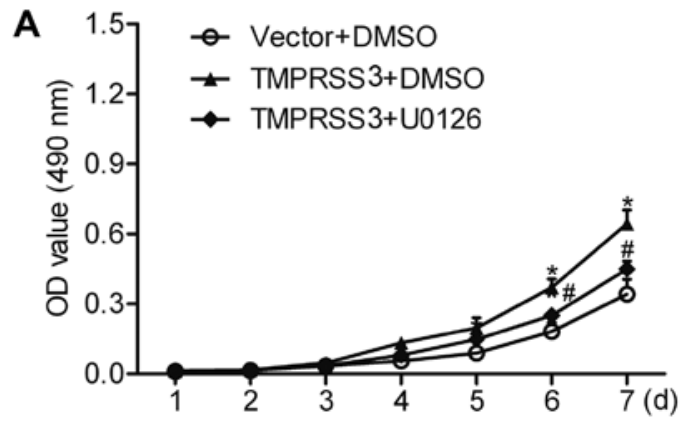

B

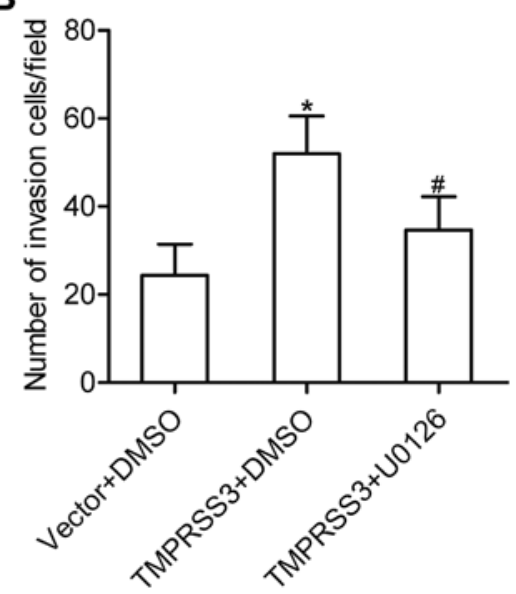

C

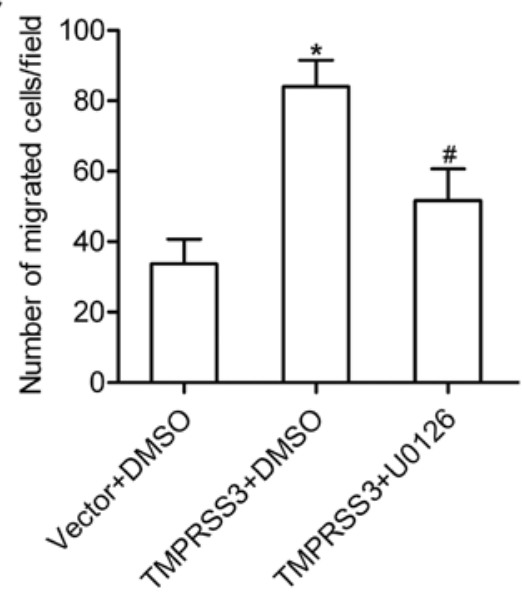

Figure 6. Effects of the ERK1/2 pathway on TMPRSS3-mediated proliferation, invasion and migration. (A) Effect of U0126 treatment on ovarian cancer cell proliferation. (B) Effect of U0126 treatment on ovarian cancer cell invasion. (C) Effect of U0126 treatment on ovarian cancer cell migration. Data are expressed as the mean $\pm \mathrm{SD}$ of three independent experiments, ${ }^{\mathrm{p}} \mathrm{p}<0.05$.

to the shControl group (Fig. 8B), indicating that TMPRSS3 contributes to the metastasis of ovarian cancer cells in vivo.

\section{Discussion}

In the present study, we found that TMPRSS3 was significantly expressed in ovarian cancer cells. To investigate the effect of TMPRSS3 expression on ovarian cancer cells, gain-or-loss of TMPRSS3 expression was introduced through ectopic overexpression or RNAi-mediated knockdown. The data showed that TMPRSS3 was able to promote the proliferation, invasion and metastasis of ovarian cancer cells. We further found that TMPRSS3 stimulated activation of ERK1/2, and the ERK1/2 pathway was involved in the biological functions of TMPRSS3. These findings suggest that TMPRSS3 promotes ovarian cancer cell proliferation, invasion and metastasis via activation of the ERK1/2 pathway.

The TMPRSS family has been reported in many tumor types $(12,13)$. Studies have found that TMPRSS3 is highly expressed in ovarian cancer $(14,15)$. In the present study, using western blotting we found that TMPRSS3 was significantly expressed in ovarian cancer cells, indicating a positive role of TMPRSS3 in ovarian cancer. It has been reported that TMPRSS4 promotes thyroid cancer proliferation via CREB phosphorylation (16), and TMPRSS4 knockdown in non-small cell lung cancer cells resulted in a significant reduction in proliferation and clonogenic capacity (17). However, the role of TMPRSS3 in cancer growth remains unknown. In the present study, we found that overexpression of TMPRSS3 enhanced the growth of ovarian cancer A2780 cells, whereas knockdown of TMPRSS3 suppressed the proliferation of ovarian cancer HO8910 cells in vitro. Moreover, knockdown of TMPRSS3 inhibited the proliferation of ovarian cancer HO8910 cells in vivo. These data suggest that TMPRSS3 contributes to the cell proliferation of ovarian cancer cells. It has been reported that TMPRSS3 expression is correlated with the metastatic potential of the clonal SUIT-2 pancreatic cancer cell line, implying that TMPRSS3 is involved in metastasis formation and tumor invasion (8). In the present study, our findings confirmed that TMPRSS3 contributed to the invasion and migration of ovarian cancer cells in vitro, and affected the metastasis of ovarian cancer cell in vivo.

Epithelial-mesenchymal transition (EMT) plays an important role in the invasion and metastasis of cancer. During the process of EMT, numerous invasion-related genes are altered. E-cadherin is a well-characterized single-pass transmembrane protein that mediates cell-cell adhesion. E-cadherin expression is usually decreased during cancer progression, and downregulation of E-cadherin is associated with tumor invasion and metastasis. Studies have found that TMPRSS4 negatively regulates E-cadherin expression in colorectal cancer and hepatocellular cancer cells $(18,19)$, and TMPRSS2 downregulates E-cadherin expression in prostate cancer cells (20). In the present study, we found that overexpression of TMPRSS3 decreased the expression of E-cadherin in A2780 cells, whereas knockdown of TMPRSS3 increased the level of 
A

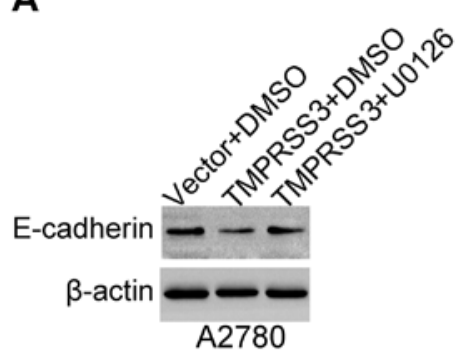

B

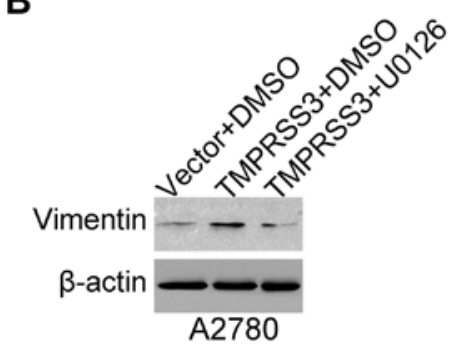

C

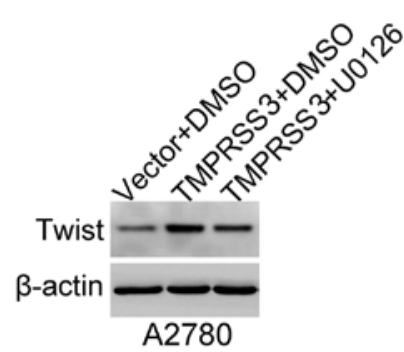

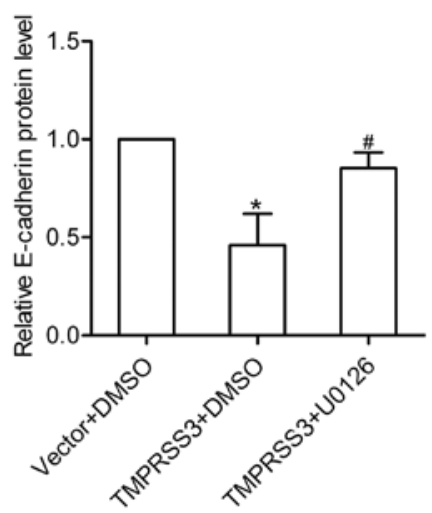
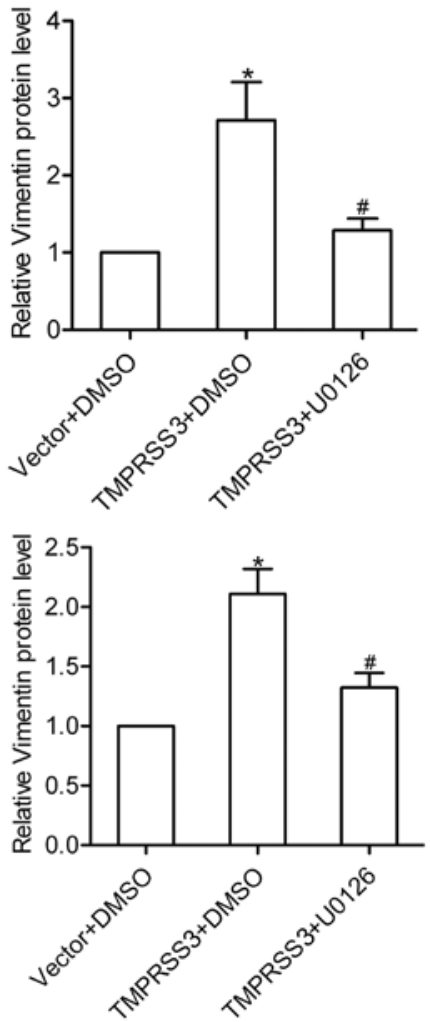

Figure 7. Effect of the ERK1/2 pathway on TMPRSS3-mediated expression of various invasion-related genes. (A) Effect of the ERK1/2 pathway on the TMPRSS3-mediated expression of E-cadherin. (B) Effect of the ERK1/2 pathway on TMPRSS3-mediated expression of vimentin. (C) Effect of the ERK1/2 pathway on the TMPRSS3-mediated expression of Twist. Data are expressed as the mean \pm SD of three independent experiments, ${ }^{*} p<0.05$.
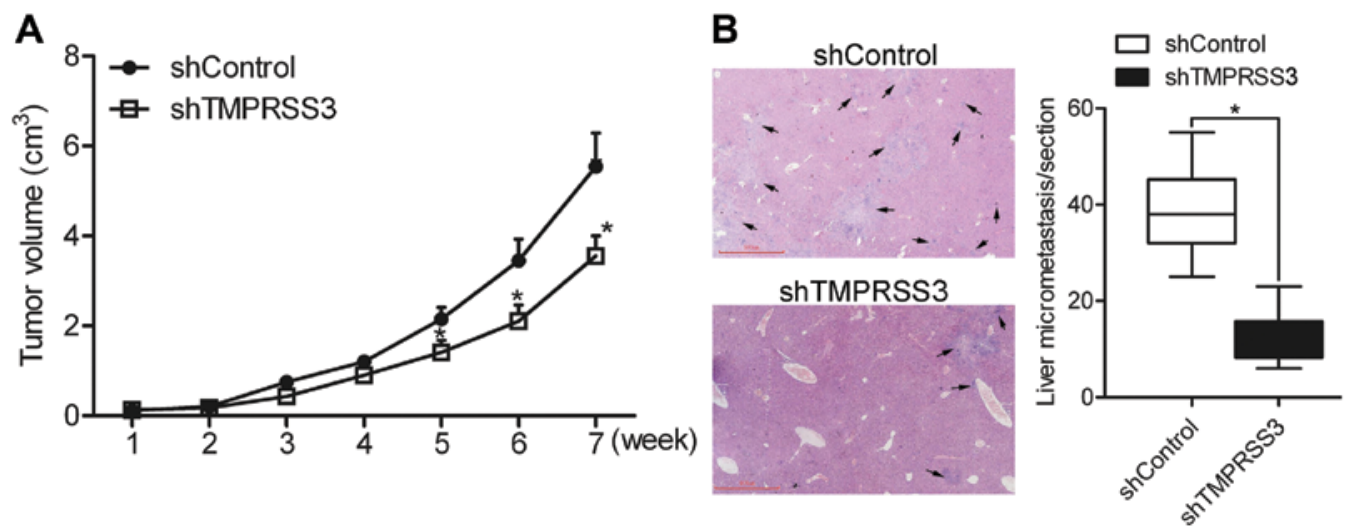

Figure 8. Effect of TMPRSS3 knockdown on the growth and metastasis of ovarian cancer cells in vivo. shControl and shTMPRSS3 cells were injected subcutaneously into two groups of nude mice $(n=8)$. (A) Tumor volume was measured every week to evaluate the effect of TMPRSS3 knockdown on the growth of HO8910 cells. (B) Eight weeks later, the mice were sacrificed. After staining with H\&E, micrometastases in the liver were observed and counted under a microscope. ${ }^{*} p<0.05$. H\&E, hematoxylin and eosin. 
E-cadherin in the HO8910 cells. Twist is a basic helix-loophelix protein that plays a role both in human development and in cancer biogenesis. Overexpression of Twist downregulates E-cadherin, which is the hallmark of EMT (21). Vimentin is the major subunit protein of the intermediate filaments of mesenchymal cells, and acts as an important hallmark of EMT (22). However, no study has reported the effect of TMPRSS3 on the expression of Twist and vimentin. In the present study, we found that TMPRSS3 positively affected the expression of Twist and vimentin in ovarian cancer cells.

It is well known that the ERK1/2 pathway plays a pivotal role in regulating tumor progression, including cell proliferation, invasion and metastasis $(23,24)$. In the present study, we found that TMPRSS3 expression induced activation of ERK1/2, suggesting that TMPRSS3 expression affects the ERK1/2 signaling pathway in ovarian cancer cells. We further found that the ERK1/2 pathway was required for TMPRSS3-mediated proliferation, invasion and migration, and participated in regulating TMPRSS3-mediated expression of E-cadherin, vimentin and Twist.

In summary, the present study demonstrated that TMPRSS3 contributes to ovarian cancer cell proliferation, invasion and metastasis, probably via activation of the ERK1/2 signaling pathway. Therefore, TMPRSS3 acts as an attractive diagnostic marker and a new target for ovarian cancer treatment.

\section{References}

1. Siegel R, Ma J, Zou Z and Jemal A: Cancer statistics, 2014. CA Cancer J Clin 64: 9-29, 2014.

2. Rodríguez Villalba S1, Díaz-Caneja Planell C and Cervera Grau JM: Current opinion in cervix carcinoma. Clin Transl Oncol 13: 378-384, 2011.

3. Roy DM and Walsh LA: Candidate prognostic markers in breast cancer: Focus on extracellular proteases and their inhibitors. Breast Cancer 6: 81-91, 2014

4. Netzel-Arnett S, Hooper JD, Szabo R, Madison EL, Quigley JP, Bugge TH and Antalis TM: Membrane anchored serine proteases: A rapidly expanding group of cell surface proteolytic enzymes with potential roles in cancer. Cancer Metastasis Rev 22: 237-258, 2003.

5. Bugge TH, Antalis TM and Wu Q: Type II transmembrane serine proteases. J Biol Chem 284: 23177-23181, 2009.

6. Hooper JD, Clements JA, Quigley JP and Antalis TM: Type II transmembrane serine proteases. Insights into an emerging class of cell surface proteolytic enzymes. J Biol Chem 276: 857-860, 2001.

7. Scott HS, Kudoh J, Wattenhofer M, Shibuya K, Berry A, Chrast R, Guipponi M, Wang J, Kawasaki K, Asakawa S, et al: Insertion of beta-satellite repeats identifies a transmembrane protease causing both congenital and childhood onset autosomal recessive deafness. Nat Genet 27: 59-63, 2001.

8. Wallrapp C, Hähnel S, Müller-Pillasch F, Burghardt B, Iwamura T, Ruthenbürger M, Lerch MM, Adler G and Gress TM: A novel transmembrane serine protease (TMPRSS3) overexpressed in pancreatic cancer. Cancer Res 60: 2602-2606, 2000.
9. Iacobuzio-Donahue CA, Ashfaq R, Maitra A, Adsay NV, Shen-Ong GL, Berg K, Hollingsworth MA, Cameron JL, Yeo CJ, Kern SE, et al: Highly expressed genes in pancreatic ductal adenocarcinomas: A comprehensive characterization and comparison of the transcription profiles obtained from three major technologies. Cancer Res 63: 8614-8622, 2003.

10. Al Saleh S, Sharaf LH and Luqmani YA: Signalling pathways involved in endocrine resistance in breast cancer and associations with epithelial to mesenchymal transition (Review). Int J Oncol 38: 1197-1217, 2011.

11. Reddy KB, Nabha SM and Atanaskova N: Role of MAP kinase in tumor progression and invasion. Cancer Metastasis Rev 22: 395-403, 2003.

12. Kebebew E, Peng M, Reiff E, Duh QY, Clark OH and McMillan A: ECM1 and TMPRSS4 are diagnostic markers of malignant thyroid neoplasms and improve the accuracy of fine needle aspiration biopsy. Ann Surg 242: 353-361, 2005.

13. Liang $\mathrm{B}, \mathrm{Wu} \mathrm{M}, \mathrm{Bu} \mathrm{Y}, \mathrm{Zhao} \mathrm{A}$ and Xie F: Prognostic value of TMPRSS4 expression in patients with breast cancer. Med Oncol 30: 497, 2013.

14. Guerrero K, Wang Z, Bachvarova M, Gregoire J, Renaud MC, Plante $M$ and Bachvarov D: A novel genome-based approach correlates TMPRSS3 overexpression in ovarian cancer with DNA hypomethylation. Gynecol Oncol 125: 720-726, 2012.

15. Zhang J, Guo H, Mi Z, Gao C, Bhattacharya S, Li J and Kuo PC: EF1A1-actin interactions alter mRNA stability to determine differential osteopontin expression in HepG2 and Hep3B cells. Exp Cell Res 315: 304-312, 2009.

16. Guan H, Liang W, Liu J, Wei G, Li H, Xiu L, Xiao H and Li Y: Transmembrane protease serine 4 promotes thyroid cancer proliferation via CREB phosphorylation. Thyroid 25: 85-94, 2015.

17. Larzabal L, Nguewa PA, Pio R, Blanco D, Sanchez B, Rodríguez MJ, Pajares MJ, Catena R, Montuenga LM and Calvo A: Overexpression of TMPRSS4 in non-small cell lung cancer is associated with poor prognosis in patients with squamous histology. Br J Cancer 105: 1608-1614, 2011.

18. Kim S, Kang HY, Nam EH, Choi MS, Zhao XF, Hong CS, Lee JW, Lee JH and Park YK: TMPRSS4 induces invasion and epithelial-mesenchymal transition through upregulation of integrin alpha5 and its signaling pathways. Carcinogenesis 31: 597-606, 2010.

19. Li T, Zeng ZC, Wang L, Qiu SJ, Zhou JW, Zhi XT, Yu HH and Tang ZY: Radiation enhances long-term metastasis potential of residual hepatocellular carcinoma in nude mice through TMPRSS4-induced epithelial-mesenchymal transition. Cancer Gene Ther 18: 617-626, 2011.

20. Leshem O, Madar S, Kogan-Sakin I, Kamer I, Goldstein I, Brosh R, Cohen Y, Jacob-Hirsch J, Ehrlich M, Ben-Sasson S, et al: TMPRSS2/ERG promotes epithelial to mesenchymal transition through the $Z E B 1 / Z E B 2$ axis in a prostate cancer model. PLoS One 6: e21650, 2011.

21. Khan MA, Chen HC, Zhang D and Fu J: Twist: A molecular target in cancer therapeutics. Tumour Biol 34: 2497-2506, 2013.

22. Satelli A and Li S: Vimentin in cancer and its potential as a molecular target for cancer therapy. Cell Mol Life Sci 68: 3033-3046, 2011.

23. Neuzillet C, Tijeras-Raballand A, de Mestier L, Cros J, Faivre S and Raymond E: MEK in cancer and cancer therapy. Pharmacol Ther 141: 160-171, 2014.

24. Schmitt JM, Abell E, Wagner A and Davare MA: ERK activation and cell growth require CaM kinases in MCF-7 breast cancer cells. Mol Cell Biochem 335: 155-171, 2010. 